\title{
Plan de negocio, proyecto constructora BellaTerra en la
}

\section{ciudad de Cuenca, Ecuador}

\section{Business plan, BellaTerra construction project in the city of Cuenca, Ecuador}

\author{
José Ricardo Goercke Villarreal. ${ }^{1}$, Juan Carlos Erazo Álvarez. ${ }^{2}$, Cecilia Ivonne Narváez \\ Zurita. $^{3}$ \& Jorge Oswaldo Quevedo Vázquez. ${ }^{4}$
}

DOI: $\underline{\text { https://doi.org/10.33262/visionariodigital.v3i2.2.629 }}$

\begin{abstract}
.
The housing deficit that currently presents the city of Cuenca, presents a business opportunity for the creation of a construction company, which allows the development of entrepreneurship, so that this research work was aimed at making a business plan that determines the feasibility of creating a construction company in the city of Cuenca. For this, a market study was conducted with a quantitative approach, with a sample of 383 people who were given a survey to determine the tastes and needs of consumers. The development of the proposal was determined by the commercial plan that included the strategic direction, economic activity and marketing mix. The technical study allowed to identify the location of the project, the layout and the definition of the good or services. On the other hand, the organizational study identified the organizational and functional structure. The financial study established that an investment of $\$ 108.653,83$ dollars is required, which will be financed with $30 \%$ of own resources and $70 \%$ through a loan through the CFN. The financial evaluation estimated a positive NPV of 62,282.63 dollars and an TIR of $51,65 \%$ which shows the feasibility of the project.
\end{abstract}

Keywords: business plan, technical study, financial evaluation

\footnotetext{
1 Universidad Católica de Cuenca, Posgradista de la Maestría en Administración de Empresas, Cuenca, Ecuador.jgoerckev@psg.ucacue.edu.ec

${ }^{2}$ Universidad Católica de Cuenca, Posgrado, Cuenca, Ecuador. jcerazo@ucacue.edu.ec

${ }^{3}$ Universidad Católica de Cuenca, Posgrado, Cuenca, Ecuador. inarvaez@ucacue.edu.ec

${ }^{4}$ Universidad Católica de Cuenca, Posgrado, Cuenca, Ecuador. joquevedov@ucacue.edu.ec
} 


\section{Resumen.}

El déficit habitacional que posee actualmente la ciudad de Cuenca, presenta una oportunidad de negocio para la creación de una empresa constructora, lo que permite el desarrollo del emprendimiento. El presente trabajo de investigación estuvo orientado a realizar un Plan de Negocios que determine la factibilidad de la creación de una empresa constructora en la ciudad de Cuenca, para lo cual, se realizó un estudio de mercado que tuvo un enfoque cuantitativo, con una muestra de 383 personas a quienes se les aplicó una encuesta para determinar los gustos y necesidades de los clientes. El desarrollo de la propuesta estuvo determinado por el plan comercial que incluyó el direccionamiento estratégico, actividad económica y marketing mix. El estudio técnico permitió identificar la localización del proyecto, el layout y la definición del bien o servicios. Por otro lado, el estudio organizacional, identificó la estructura organizacional y funcional. El estudio financiero estableció que se requiere de una inversión de $\$ 108.653,83$ dólares, que será financiado con un 30\% de recursos propios y el 70\% mediante un crédito a través de la CFN. La evaluación financiera identificó un VAN positivo de $\$ 62.282,63$ dólares y una TIR del 51,65\% que muestra la factibilidad del proyecto.

Palabras claves: Plan de negocio, estudio técnico, evaluación financiera

\section{Introducción.}

El sector de la construcción siempre ha tenido un papel influyente en la economía ecuatoriana, tanto del sector privado y mucho más del sector público, por el modelo institucionalizado actualmente. Sin embargo, este tuvo un declive por varios factores que perjudicaron la economía del país.

El dinamismo de la construcción se vio afectado, pues tiene una relación directa con la inversión en obras públicas, así como en la oferta y demanda de vivienda, que se ha contraído por los bajos ingresos del Estado y la contracción en los ingresos de los hogares. De acuerdo a cifras provisionales del Banco Central de Ecuador, este sector fue el de mayor reducción en términos de PIB (-10,3\%) en el 2016. (Mundo Constructor, 2017, p. 12)

En este mismo sentido, con la recuperación prevista para el 2018 y la decisión política del actual gobierno nacional de fomentar la inversión privada nacional y extranjera, tuvo como meta "incrementar de 53\% a 95\% el número de hogares con vivienda propia y digna que se encuentran en situación de extrema pobreza a 2021" (SENPLADES, 2017). Según él (INEC, 2012) "los costos de viviendas varían dependiendo de varios factores cuyos costos van desde 25 mil hasta cientos de miles de dólares según la oferta inmobiliaria actual; su financiamiento se lo calcula dependiendo de los ingresos de las familias" (p.6). 


\section{Empresa constructora en la ciudad de Cuenca}

La ciudad de Cuenca es la tercera ciudad más importante del país por su desarrollo y nivel económico motivo por el cual resulta interesante y una oportunidad de evaluar y aprovechar la demanda potencial de vivienda a través de la creación de una empresa que se dedique a la construcción y comercialización de las mismas, para ello se fijó como objetivo general realizar un Plan de Negocios que determine la factibilidad de la creación de una empresa constructora en la ciudad de Cuenca, a través del cumplimiento de objetivos específicos como; fundamentar teóricamente el proyecto, evaluar la factibilidad de la creación de una empresa constructora, elaborar los estudios del plan de negocio y validar la propuesta mediante método de expertos.

Además, entre las variables determinadas se estableció como variable independiente el plan de negocios y como variable dependiente la factibilidad. En relación a ello, el plan de negocios se alimenta del estudio de factibilidad, ya que, este tiene como finalidad de investigar y descubrir si la idea de negocio es realmente viable, y si esta resulta positiva se la presentará a los inversionistas y a su vez garantizará al empresario ejecutarla con alto factor de éxito.

\section{Plan de negocio}

El proyecto es un instrumento de labor para todo individuo que desea desarrollar e iniciar una nueva empresa. Es una herramienta escrita que analiza, describe e identifica una ocasión de comercio, pudiendo surgir en esta etapa asuntos definitivos en los que primeramente no se remedió Almoguera (2013).

Existen diferentes mercados en los que se puede desarrollar un negocio. En la creación de un negocio, se busca satisfacer una necesidad mediante un producto o servicio. En la actualidad, existen varios factores que se deben tomar en cuenta al ingresar en un mercado ya establecido (Romo, 2013).

Entre las características más relevantes de un plan de negocio de acuerdo a Díaz de Santos (2015) las principales características de un buen plan de negocios son:

- Lógico: Su elaboración responde a planeamientos racionales.

- Progresivo: Cada parte depende de la anterior siguiendo una "cadena" en secuencia

- Realista: Constituye un documento eminentemente práctico, basado en la "realidad" de la empresa y sus potencialidades.

- Coherente: Todas las partes de un plan de negocios deben ser consistentes y armónicas entre sí.

- Orientado a la acción: Todas las partes de un plan de negocios deben conducir a la ejecución de acciones específicas, claras y precisas. 
Para la formulación del plan de negocios es necesario considerar todos los aspectos posibles y seguir una metodología estructurada técnicamente, además de considerar varios escenarios que diagnostiquen la situación actual donde la nueva empresa va a incursionar, dichos componentes son: mercado, requerimientos legales, gestión administrativa, técnica y financiera.

\section{Componentes del plan de negocio}

\section{- Estudio de mercado}

En relación al estudio de mercado, para Operagb (2017) este análisis "indaga como el mercado se comporta ante el servicio o producto, con el propósito de planificar la maniobra productiva más conveniente" (p.15). Dicho estudio de mercado se compone de la siguiente manera: mercado, clientes, la competencia y las fuentes de información; cada una de estos elementos de describen a continuación:

El estudio de mercado es una noción que puede parecer aterradora. En su propósito de generación de la empresa, muchos se desaniman incluso antes de haber investigado para saber qué obtendrán a cambio. El estudio de mercado está conformado por la identificación del mercado (tamaño, cuota, estructura del mercado, mercado meta), clientes, competencia y las fuentes de información.

\section{Tamaño del mercado}

Según Parmerlee (2012) indica que:

El propósito de intentar predecir el tamaño del mercado es decidir cómo se insertará en ese mercado a partir de sus acciones de marketing. El tamaño del mercado se define en cuatro niveles; los primeros dos niveles definen el mercado y los otros dos definen su capacidad de venta en el mismo. (p. 2)

Entre los niveles que se mencionan en el tamaño de mercado se detallan a continuación:

- El nivel uno es el potencial del mercado; su propósito es establecer la máxima venta posible de una categoría de producto, en moneda corriente o en unidades, para la totalidad de las empresas.

- El nivel dos es el pronóstico de mercado; su propósito es establecer las ventas estimadas de una categoría de producto, en moneda corriente o en unidades, de cada una de las empresas presentes dentro de una zona específica y un periodo de tiempo definido.

- El nivel tres es el potencial de ventas; el propósito es establecer la máxima cantidad de producto que, en moneda corriente o unidades de venta, la compañía será capaz de producir y vender y para la cual podrá ofrecer servicio adecuado cada año.

- El nivel cuatro es el pronóstico de ventas: su propósito es predecir las ventas estimadas, en moneda corriente o en unidades, de las líneas de producto para ese ejercicio. 


\section{Estructura del mercado}

La empresa está orientada a ejercicios comerciales dirigidos a la competencia. El mercado objetivo está combinado por el conjunto de clientes viables que se pretenden transformar en compradores según lo manifestado por (Operagb, 2017).

\section{Clientes}

Según lo manifestado por Rapahmell (2017) define a clientes como "el factor principal de todo negocio, es por ello que las organizaciones le deben presentar mucha atención, el éxito de una empresa radica en la demanda de sus clientes siendo ellos los verdaderos protagonistas del desarrollo de la organización" (p. 53). Un cliente satisfecho volverá y se comunicará con la empresa. No obstante, un comprador descontento desprestigiara la empresa, en sí, comunicará a sus allegados su insatisfacción y no regresara al negocio.

\section{La competencia}

Según Zevallos (2016) para que el gerente pueda dirigir mejor la organización y la empresa debe poseer las siguientes ventajas:

1. Conocer el mercado y el producto

2. Identificar el tipo de competencia

3. Formar o reformar la organización

4. Evaluar y comprobar lo realizado con lo planeado

5. Reaccionar rápidamente ante los hechos

\section{Fuentes de información}

Existen dos tipos de fuentes de información estas pueden ser primarias y secundarias: las primarias son información que resuelve problemas determinados, no constando inicialmente datos, este tipo de fuentes, generan, consiguen datos únicos, y, las fuentes secundarias, están cimentados en datos ya históricos: se trata sólo de mejorar una búsqueda existente. La particularidad habitual es que manejan datos ciertos. Se usa en si datos existentes Ferre (2014).

\section{- Estudio técnico}

La complejidad de los proyectos es muy variada dependiendo de la dificultad de los mismos, por lo que se recurrió a un mayor número de expertos técnicos para el diseño de la inversión inicial necesaria. Acudir en estos casos a profesionales con solvencia (ingenieros, arquitectos, abogados, etc.) es una estrategia necesaria que requiere cierta atención y detenimiento. 
Según Hamilyon y Pezo (2015) las partes que conforman un estudio técnico se describen de la siguiente manera:

- Planos del conjunto y de detalle para que los trabajos a realizar queden definidos y delimitados correctamente.

- Un pliego de prescripciones técnicas donde se describen los trabajos a realizar y su ejecución. Un elemento importante es una descripción de cómo se llevará a cabo la ejecución, mediciones, revisión de particularidad, así como una especificación de las distintas obligaciones de los actuantes en los trabajos,

- Presupuesto. Expresión detallada de las partes con especificación de los precios unitarios, así como todo lo que es objeto de valoración.

- Programa de desarrollo de los trabajos o plan de trabajo que incluya una previsión de tiempos y costos reales, pues en ocasiones no se tienen en cuenta acontecimientos imprevistos que pueden introducir retrasos en la ejecución de los trabajos.

- Información diversa que por motivos de una legislación concreta haya que aportar.

- Estudio de seguridad y salud. (p.6)

\section{- Estudio administrativo y legal}

Así también, el estudio administrativo y legal "se refiere al estudio del marco administrativo y legal del proyecto es decir la estructura organizativa y los requerimientos legales del proyecto" (Martínez, 2015, p. 36).

\section{Estudio legal}

La posibilidad legal de un plan establece la presencia o escases de reglas que lograran limitar la ejecución del emprendimiento o estipular su realización al desempeño de algunas obligaciones mínimas para poder efectuarlo Martínez (2015). El análisis legal de la posibilidad financiera valorará las consecuencias que los estatutos establecidos adquirirán sobre los beneficios y costos de un plan que ya es factible reglamentariamente. Dentro del estudio legal según Almoguera (2013) es necesario tomar en cuenta los siguientes elementos normativos:

- Permisos y patentes locales

- Producción de tratados comerciales y laborales.

- Análisis de propiedad y presencia y títulos de posesión

- Consumos relacionados con la inscripción en registros legales de pertenencia

- Empadronamiento de marcas

- Permisos y aranceles de importación

- Compensaciones de despidos

- Convenciones compañías de seguro laboral

- Deberes en caso de incidentes laborales

- Amortizaciones contables y procedimiento fiscal de devaluaciones. 
- Tributaciones al valor agregado, la propiedad y las ganancias.

- Regulaciones internacionales.

\section{Estudio administrativo}

Trata de instituir la estructura empresarial que administrará el proyecto, trazado aquella que más se aplique a las exigencias. El estudio reside en establecer los puntos organizativos que corresponderá generar una nueva empresa para su entidad como ejemplo su planeación indispensable, su estructura comunitaria, sus puntos fiscales, legales, laborales, la creación de las fuentes y metodologías de reclutamiento, el proceso de clasificación y la persuasión que se dará a los trabajadores requerirles para su legalización (Baca, 2014).

Dentro del estudio administrativo es importante desarrollar varias características, con el fin de direccionar las funciones, responsabilidades y obligaciones de los recursos humano:

- Organigrama: el organigrama personifica la organización formal de una distribución. Es una figura combinada por una serie de cuadriláteros, que simbolizan a las asociaciones, los mismos que se juntan entre sí por rayas, que interpretan a las analogías de comunicación y atribución al interior de la empresa (Gilli, 2017).

- Manual de funciones: señala que tiene como objetivo primordial resaltar la estructura empresarial las funciones, responsabilidades y relaciones de todas las dependencias al interior de la organización debe ser estimado como una herramienta eficiente sumiso a permutaciones que ocurren de las insuficiencias propias de cada negocio, y de la investigación técnica constante para conservar su ventaja. (Martínez, 2015).

\section{Estudio financiero}

Según Guzmán (2016) indica que "el estudio económico financiero de un proyecto se ha agrupado en tres aspectos las inversiones de los presupuestos de ingresos, costos y el financiamiento" (p.107).

El estudio económico financiero sienta las bases para saber cuál será el costo de inversión de la empresa, para conocer si es posible o no la ejecución de esta. Para ello, se calcularán los estados de resultados, los ingresos, los costos, punto de equilibrio, y balance general.

Estado de resultados: muestra para un periodo dado, las entradas de la organización sus salidas en conocimiento de precios. Si la discrepancia entre entradas menos precio es efectiva, la organización consigue beneficios, no obstante, si este es negativo la asociación alcanza desventajas (Fernández, 2013).

Ingreso: es la entrada o percepción de dinero en forma de efectivo, balances por recolectar $\mathrm{u}$ otro activo que se recogen de los usuarios por impulso de la asistencia de un trabajo, la venta de un producto o de inversiones en valores, (Brock y Palmer, 2016). 
Gasto: Es el costo que ocasiona el manejo de los servicios o cosas con el propósito de generar entradas. No toda inversión es un consumo, ya que puede prorrogar en magnitud y tiempo, (Brock y Palmer, 2016).

Punto de equilibrio: se basa en que todos los costos asociados con la generación de un servicio o producto específico pueden dividirse en dos categorías: costos variables y costos fijos (Jurado, 2016). El punto donde se intercepta la línea de los costos generales con la línea de las entradas por comercializaciones, se conoce como el punto de equilibrio, por este método el punto de equilibrio se expresa en unidades.

Balance general: Horngren, Sundem y Elliott (2016) indican que a un lado se sitúan los activos, que personifica los recursos de la asociación. Al otro lado coloca capitales, que simbolizan las fuentes de los recursos disponibles para adquirir el activo.

\section{Evaluación económica}

La formulación y evaluación de un proyecto de inversión implica una secuencia de acciones o aproximaciones sucesivas para determinar la factibilidad de cada característica como condición para continuar con el desarrollo y avance de otros aspectos del proyecto de inversión.

En efecto, las particularidades de mercado y comercialización constituyen una evaluación sobre los elementos sustantivos del mismo, la caracterización del bien, el segmento de mercado, el comportamiento del consumidor, el comportamiento de la oferta, la estructura prevaleciente en el mercado, el comportamiento de los precios, la comercialización, la existencia y cuantificación de una demanda insatisfecha, etc. Algo similar ocurre con los capítulos de carácter técnico y los de tipo jurídico administrativo, donde se plantean una serie de alternativas que son evaluadas y analizadas, a fin de poder determinar su factibilidad técnica administrativa y jurídica, (Gallardo, 2017).

En contexto, un plan de negocios es un documento escrito que describe en detalle cómo una empresa, generalmente una nueva, va a lograr sus objetivos, establece un plan escrito desde un punto de vista de marketing, financiero y operativo.

\section{Metodología}

Para poder obtener la información más relevante en el desarrollo de esta investigación fue necesario establecer un diseño de investigación que determinó que este estudio tuvo un enfoque cuantitativo, mediante la aplicación de mediciones objetivas aplicando el análisis estadístico de los datos que fueron recolectados a través la aplicación de técnicas de obtención de datos, para luego, mediante la aplicación de métodos estadísticos, reunir datos numéricos y generalizarlos entre grupos de personas. Así también, se abordó una 
investigación de carácter descriptiva, que sirvió para analizar las características del fenómeno en estudio, sobre la factibilidad de establecer una empresa constructora en la ciudad de Cuenca.

Los métodos utilizados en esta investigación fueron: inductivo - deductivo que permitió el razonamiento de las principales teorías, con el propósito de identificar algunas evidencias, por otra parte, se usó el método sistemático que generó un sentido común, considerando que la industria de la construcción es sistémico en sus componentes y realidades; y el método histórico - lógico que facilitó la obtención de información de fuentes confiables, estructuradas de una manera cronológica y lógica, para establecer los antecedentes de investigación.

Se aplicó una encuesta, que es una de las técnicas más conocidas y utilizadas por los investigadores de mercados, para obtener información precisa. Para la aplicación de la misma se consideró al total de habitantes de la ciudad de Cuenca, conforme al VII Censo de Población y Vivienda del año 2010, que es de 331.888 habitantes (158.365 hombres y 173.523 mujeres). De estos se tomó en consideración a las personas que pertenecen a la Población Económicamente Activa (PEA), entre el rango de edad de entre los 20 a los 64 años de edad, puesto que de acuerdo a la Encuesta Nacional de Empleo, Desempleo y Subempleo (ENEMDU), estas personas se caracterizan por tener un empleo o una actividad económica más estable, donde por lo general el padre y la madre trabajan, disponen de los ingresos suficientes para mantener un hogar.

En este contexto, la población estuvo conformada por 189.316 habitantes, para lo que, fue necesario extraer una muestra de la población total para obtener información más precisa a través de la encuesta, para ello se aplicó a 383 habitantes de la ciudad de Cuenca y del mismo se obtuvieron los siguientes resultados:

El 64\% de los encuestados buscan adquirir una casa, el $18 \%$ un departamento, el 9,1\% una oficina, el $7.8 \%$ un local comercial y el $1 \%$ otros tipos de inmueble (generalmente terrenos o edificios). El tipo de inmueble que busca una persona está relacionado principalmente con los gustos del cliente, capacidad económica y el tipo de actividad económica, considerando que en el mercado se encuentra una diversidad de inmuebles que se acoplan a cada necesidad. Antes de adquirir algún tipo de inmueble el cliente toma en cuenta el precio (41\%), luego la localización (24\%), después al tipo de financiamiento $(18,8 \%)$, la plusvalía $(14,1 \%)$ y finalmente el $1 \%$ a la accesibilidad.

Además, en el mercado inmobiliario se conjugan una serie de factores que intervienen en los procesos de comercialización, entre los que se puede resaltar el Asesoramiento Inmobiliario, que generalmente se lo realiza mediante una empresa legalmente constituida, brindando confiabilidad y validez al proceso de negociación de cualquier tipo de inmueble, en este 
sentido, de acuerdo con los encuestados un 78,07,\% si ha utilizado los servicios de un Asesor Inmobiliario, mientras el $21,9 \%$ no lo ha hecho.

También, se pudo constatar que, de existir una nueva empresa que brinde los servicios inmobiliarios, la mayor parte de las personas $(77 \%)$ harán uso de sus servicios. En referencia a los resultados del estudio de mercado, se pudo establecer el perfil del cliente, determinando necesidades y requerimientos que permitirán establecer lineamientos para diseñar un plan de negocio para la creación de una empresa constructora en la ciudad de Cuenca.

\section{Resultados}

La industria de la construcción está altamente diversificada e involucra a numerosos clientes como constructores de propiedades, promotores inmobiliarios, proveedores de materiales y contratistas. En este sentido, la creación de una empresa constructora aportaría al desarrollo económico de la ciudad de Cuenca, puesto que esta industria hace su contribución directa al crecimiento económico, puesto que proporciona la base la producción y distribución de bienes y servicios.

Para lograr esta meta, se requiere de la elaboración de un plan de negocios, que es una herramienta estratégica muy importante para los empresarios, puesto que un buen plan de negocios no solo ayuda a enfocarse en los pasos específicos necesarios para que las ideas de negocios tengan éxito, sino que también ayuda a lograr objetivos a corto, mediano y largo plazo, este plan consta de las siguientes etapas:

Figura 1. Esquema de la propuesta

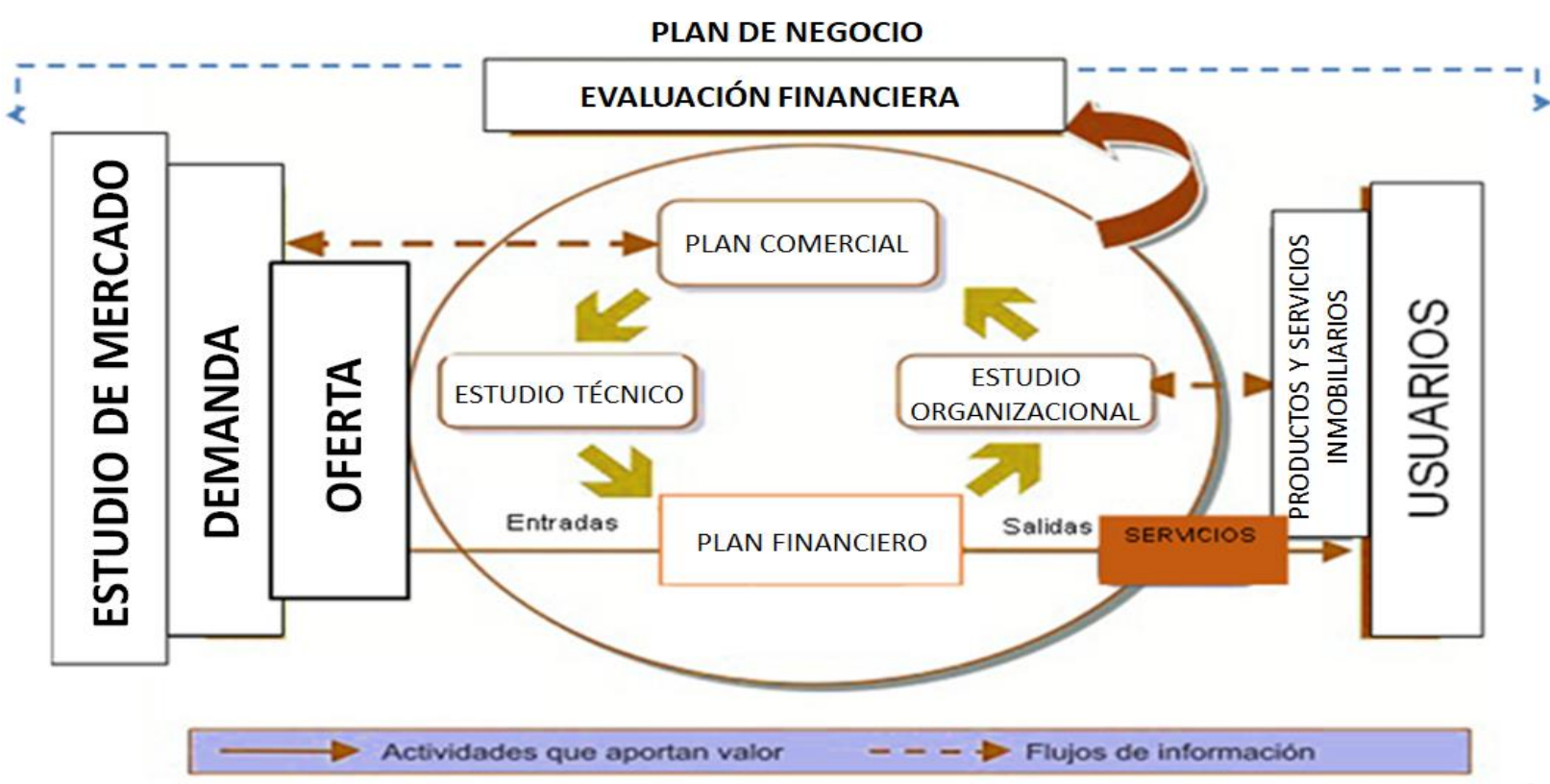




\section{Primera etapa: Estudio de mercado}

En el estudio de mercado se determina la oferta y la demanda dentro del sector inmobiliario. Actualmente las propiedades varían entre \$ 100.000 y \$200.0000, no obstante, los proyectos de Vivienda de Interés Público (VIP) ofertan casas de \$70.000 a \$90.000. Más de 100 empresas ofertan inmuebles de rango social, medio y de gama alta a nivel nacional según (BIAU, 2019).

Para el presente proyecto se han definido más de 25 empresas que se dedican a la construcción de viviendas en la ciudad de Cuenca como parte de la oferta, no obstante, la Constructora "BELLATERRA" ofertará un total de 10 casas por año a un valor estimado de $\$ 63.908,99$.

\section{Segunda etapa: Estudio técnico}

Mediante el estudio técnico se determinó que el proyecto estará ubicado en la provincia del Azuay, cantón Cuenca, Parroquia San Joaquín, en la Avenida Arizaga Toral y Pimienta. El layout de la empresa se diseñó considerando cada una de las áreas funcionales, tales como gerencia, contabilidad, ventas, recepción.

Figura 1. Layout de la empresa

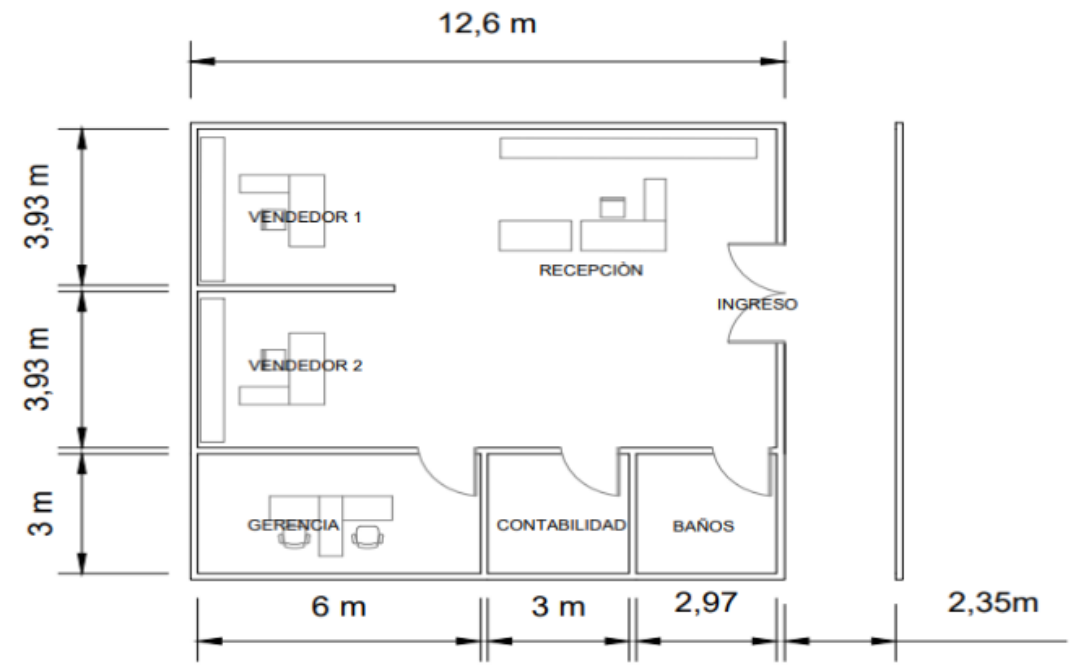

Para la definición del bien o servicio, se consideran los procesos productivos, como se muestra a continuación: 
Figura 3. Proceso productivo

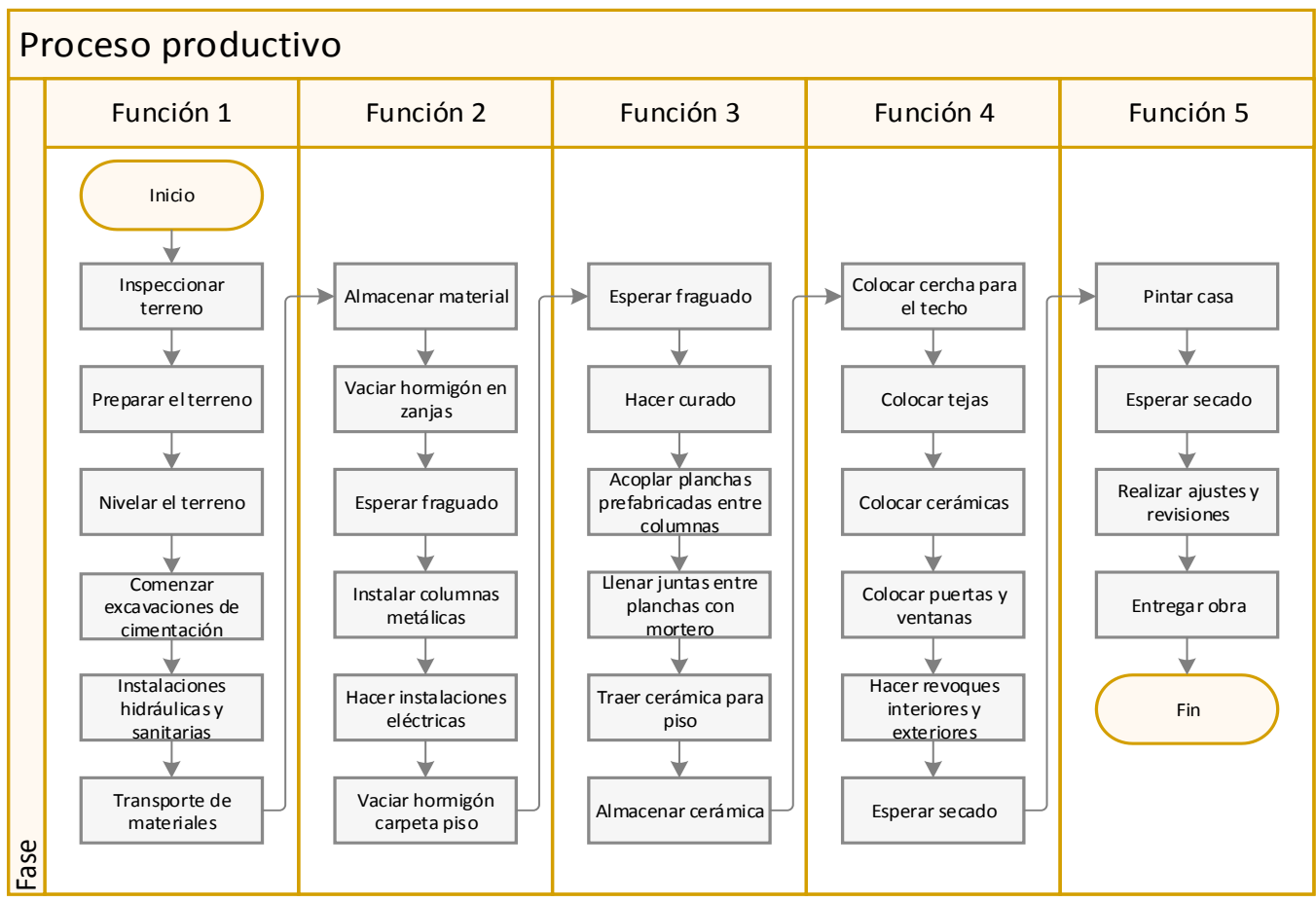

Fuente: Datos obtenidos de la Cámara de la Construcción de Cuenca (2019)

\section{Tercera etapa: Plan comercial}

En relación a la etapa del plan comercial, se define que la empresa a crearse se denominará Constructora "BELLATERRA" que de acuerdo al Código CIIU 4.0, la empresa estará en la actividad denominada "F4100.10: Construcción de todo tipo de edificios residenciales: casas familiares individuales, edificios multifamiliares, incluso edificios de alturas elevadas, viviendas para personas de la tercera edad, casas para beneficencia, orfanatos, cárceles, cuarteles, conventos, casas religiosas. Incluye remodelación o rehabilitación de estructuras existentes". Esta empresa se fijará una misión, visión y valores que se desarrollan a continuación:

Misión: Empresa que combina experiencia e innovación en la creación, diseño y construcción proyectos residenciales y comerciales, trabajando bajo técnicas y tecnología de vanguardia con los más altos estándares de calidad internacional.

Visión: Para el año 2022 convertirse en la empresa constructora preferida en la ciudad de Cuenca, reconocida por su alto nivel de calidad las actividades creación, diseño y construcción proyectos residenciales y comerciales. 
Valores:

- Excelencia

- Trabajo en equipo

- Integridad

- Compromiso

- Sostenibilidad

- Responsabilidad

Por otra parte, para establecer un adecuado plan comercial se determinó el Marketing Mix, es decir se fijó estrategias de precio, producto, promoción y plaza como muestra a continuación:

\section{- Precio}

Negociar adecuadamente con los proveedores para alcanzar los mejores precios en la adquisición de insumos, materiales y mano de obra. El precio está determinado por la necesidad de cubrir los costos operativos, administrativos y comerciales, considerando también los precios establecidos en la competencia y un margen de utilidad adecuado para los inversionistas.

\section{- Producto}

El logotipo que refleja los productos y servicios de la empresa constructora, se muestra en la siguiente figura:

Figura 4. Logotipo de la empresa

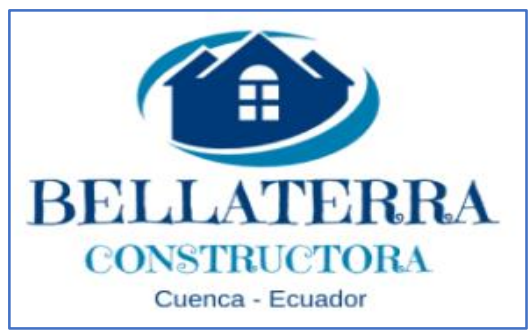

La estrategia de producto de la empresa, será el implementar un sistema propio de la empresa para el desarrollo y la ejecución de los proyectos inmobiliarios. Además, se establecerá como otra estrategia y valor agregado la construcción de viviendas innovadoras en relación a la volumetría y en fachada, utilizando dos tipos de madera como son: Teka y Chonta, acorde al tipo de vivienda que solicita el cliente y que está actualmente en el mercado. 
En contexto, el producto que ofertará la Constructora Bellaterra, no solo consistirá en satisfacer las necesidades de los clientes con la edificación de su vivienda acorde a los requerimientos, sino también, se plantea una idea innovadora en relación a la utilización de materiales para la construcción como se indicó en el párrafo anterior.

\section{- Promoción y publicidad}

La venta de los proyectos se los iniciará mediante planos u obra gris. Se buscará que la promoción y publicidad del proyecto se lo realice a través de instituciones financieras que brinden altas posibilidades para la compra de vivienda con facilidades para los clientes.

\section{- Plaza}

Identificar permanentemente áreas de construcción en diferentes sectores de la ciudad, para desarrollar nuevos proyectos y expandir el negocio.

\section{Cuarta etapa: Estudio organizacional}

En el estudio organizacional se estableció que la estructura organizacional estará acoplada a las necesidades del cliente y las partes funcionales que requiere la organización. Se determinó que la constructora tendrá: un gerente general, dentro del área administrativa se contratan un contador y un auxiliar, en el área de proyectos inmobiliarios se encontrará dos personas que se encarguen de la investigación y desarrollo, la construcción de proyectos; y el área comercial estará formada por una persona de ventas y post venta. Es importante mencionar que se elaboró un manual de funciones para las principales funciones de las áreas organizacionales de la empresa

\section{Quinta etapa: Estudio financiero}

En relación a la parte financiera del proyecto se prevé una inversión total de $\$ 108.653,83$. No obstante, se buscará el financiamiento de la inversión, a través de un crédito de la Corporación Financiera Nacional, para cubrir hasta el 70\% del total de la inversión, con cuotas semestrales y dos de gracia, a una tasa de interés del 9,33\%. El otro 30\% será financiado con recursos propios del inversionista.

\section{- Costos y gastos}


Tabla 1. Presupuesto de costos y gastos

\begin{tabular}{|c|c|c|c|c|c|}
\hline \multirow{2}{*}{ Detalle } & \multicolumn{5}{|c|}{ Años } \\
\hline & 1 & 2 & 3 & 4 & 5 \\
\hline \multicolumn{6}{|l|}{ Costo primo } \\
\hline Materia prima directa & $491.607,65$ & $492.689,19$ & $493.773,10$ & $494.859,41$ & $495.948,10$ \\
\hline Total costo primo & 491.607,65 & 492.689,19 & $493.773,10$ & 494.859,41 & $495.948,10$ \\
\hline \multicolumn{6}{|l|}{ Gastos de operación } \\
\hline \multicolumn{6}{|l|}{ Ventas } \\
\hline Sueldos & $19.337,60$ & $19.380,14$ & $19.422,78$ & $19.465,51$ & $19.508,33$ \\
\hline Publicidad & 800,00 & 801,76 & 803,52 & 805,29 & 807,06 \\
\hline Total gastos ventas & $20.137,60$ & 20.181,90 & $20.226,30$ & $20.270,80$ & $20.315,40$ \\
\hline \multicolumn{6}{|l|}{ Administrativos } \\
\hline Sueldos & $35.962,50$ & $36.041,62$ & $36.120,91$ & $36.200,38$ & $36.280,02$ \\
\hline Arriendos & $6.000,00$ & $6.013,20$ & $6.026,43$ & $6.039,69$ & $6.052,97$ \\
\hline Servicios básicos & $3.600,00$ & $3.607,92$ & $3.615,86$ & $3.623,81$ & $3.631,78$ \\
\hline Materiales de oficina & 439,20 & 440,17 & 441,13 & 442,11 & 443,08 \\
\hline Materiales de aseo & 996,00 & 998,19 & $1.000,39$ & $1.002,59$ & $1.004,79$ \\
\hline Amortización & $1.200,00$ & $1.200,00$ & $1.200,00$ & $1.200,00$ & $1.200,00$ \\
\hline Depreciaciones & $1.542,80$ & $1.542,80$ & $1.542,80$ & $1.542,80$ & $1.542,80$ \\
\hline Total gastos administrativos & 49.740,50 & $49.843,89$ & 49.947,52 & $50.051,37$ & $50.155,45$ \\
\hline \multicolumn{6}{|l|}{ Financieros } \\
\hline Intereses préstamos & $7.025,55$ & $6.073,87$ & $4.804,90$ & $3.413,33$ & $1.887,31$ \\
\hline Total gastos financieros & $7.025,55$ & $6.073,87$ & $4.804,90$ & $3.413,33$ & $1.887,31$ \\
\hline Total gastos de operación & $76.903,65$ & 76.099,66 & $74.978,72$ & 73.735,50 & $72.358,16$ \\
\hline Total & $568.511,31$ & $568.788,85$ & $568.751,82$ & $568.594,90$ & $568.306,25$ \\
\hline
\end{tabular}

\section{- Ingresos}

En relación a los ingresos, estos están proyectados en la construcción y venta de un proyecto con 10 unidades habitacionales por año, cuyo precio ha sido proyectado de acuerdo a la tasa de inflación del año 2018 (0,22\%), de la siguiente manera:

Tabla 2. Proyección de ingresos totales

\begin{tabular}{|c|c|c|c|c|c|c|}
\hline Año & Tipo & Unidades & $\begin{array}{l}\text { Costos de } \\
\text { producción }\end{array}$ & Utilidad & $\begin{array}{l}\text { Precio de } \\
\text { Venta }\end{array}$ & Ingresos \\
\hline $\begin{array}{l}\text { Proyecto } \\
\text { (año 1) }\end{array}$ & $\begin{array}{l}\text { Casa de } 75 \text { m2 } \text { de } \\
\text { construcción: } 2 \text { pisos, } 3 \\
\text { dormitorios, } 2 \text { baños, sala } \\
\text { comedora, cocina }\end{array}$ & 10 & $49.160,77$ & $14.748,23$ & $63.908,99$ & $639.089,95$ \\
\hline $\begin{array}{l}\text { Proyecto } \\
\text { (año 2) }\end{array}$ & $\begin{array}{l}\text { Casa de } 75 \text { m2 de } \\
\text { construcción: } 2 \text { pisos, } 3 \\
\text { dormitorios, } 2 \text { baños, sala } \\
\text { comedora, cocina }\end{array}$ & 10 & $49.268,92$ & $14.780,68$ & $64.049,59$ & $640.495,94$ \\
\hline $\begin{array}{l}\text { Proyecto } \\
\text { (año 3) }\end{array}$ & $\begin{array}{l}\text { Casa de } 75 \text { m2 de } \\
\text { construcción: } 2 \text { pisos, } 3 \\
\text { dormitorios, } 2 \text { baños, sala } \\
\text { comedora, cocina }\end{array}$ & 10 & $49.377,31$ & $14.813,19$ & $64.190,50$ & $641.905,04$ \\
\hline $\begin{array}{l}\text { Proyecto } \\
\text { (año 4) }\end{array}$ & $\begin{array}{l}\text { Casa de } 75 \text { m2 de } \\
\text { construcción: } 2 \text { pisos, } 3 \\
\text { dormitorios, } 2 \text { baños, sala } \\
\text { comedora, cocina }\end{array}$ & 10 & $49.485,94$ & $14.845,78$ & $64.331,72$ & $643.317,23$ \\
\hline $\begin{array}{l}\text { Proyecto } \\
\text { (año 5) }\end{array}$ & $\begin{array}{l}\text { Casa de } 75 \text { m2 de } \\
\text { construcción: } 2 \text { pisos, } 3 \\
\text { dormitorios, } 2 \text { baños, sala } \\
\text { comedora, cocina }\end{array}$ & 10 & $49.594,81$ & $14.878,44$ & $64.473,25$ & $644.732,53$ \\
\hline
\end{tabular}


- Evaluación financiera

\section{Punto de equilibrio}

Así también, el punto de equilibrio se producirá cuando la empresa trabaja a una capacidad del 53,33\% y tiene ventas por 340.828,07 dólares.

\section{Valor Actual Neto}

Para la determinación del Valor actual neto se realizó el siguiente calculo:

Factor de Actualización $=\frac{1}{(1+\mathrm{i})^{\mathrm{n}}}$

VAN $=\quad \Sigma$ FNA - INVERSIÓN INICIAL

VAN $=230.444,91-108.653,83$

$\mathbf{V A N}=121.791,09$

\section{Tasa Interna de Retorno}

$\mathrm{TIR}=\mathrm{Tm}+\mathrm{Dt} \frac{(\text { VAN menor })}{(\text { VAN menor }- \text { VAN mayor })}$

$\mathrm{TIR}=51+1\left(\frac{1.052,81}{1.621,49}\right)$

TIR $=51,65 \%$

Es imprescindible indicar que los indicadores financieros son favorables para los inversionistas, puesto que, según los criterios de decisión el VAN es mayor que cero y por ende se acepta la inversión, al igual que el valor de la TIR también es aceptable para ejecutar la inversión en la creación de la empresa Constructora "BELLATERRA".

\section{Conclusiones}

Se concluye que existe una necesidad de crear una empresa constructora, ya que de acuerdo a datos emitidos por organismos oficiales actualmente se presenta un déficit creciente de viviendas, que presenta la necesidad de que se establezca este tipo de negocio. Mediante el estado del arte se pudo establecer un fundamento teórico que facilitó la comprensión de diversos términos conceptuales además de identificar los pasos que se requieren para establecer la factibilidad del proyecto.

El estudio de mercado tuvo un enfoque cuantitativo, mediante un estudio descriptivo. Para la determinación de la población se consideró al total de la población de la ciudad de Cuenca, que de acuerdo al INEC es de 331.888 habitantes. De estos se identificó a las personas que pertenecen a la PEA, entre los 20 a los 64 años de edad, reflejando una cantidad de 189.316 
habitantes. La aplicación de la fórmula de la muestra determinó un total de 383 personas a quienes se les aplicó la encuesta.

El desarrollo de la propuesta se determinó por el plan comercial que incluyó el direccionamiento estratégico, actividad económica y marketing mix. El estudio técnico permitió identificar la localización del proyecto, el diseño de la planta (layout) y la definición del bien o servicios. Por otro lado, el estudio organizacional, identificó la estructura organizacional y funcional. El estudio financiero determinó que se requiere de una inversión de $\$ 108.653,83$ dólares, que será financiado con un $30 \%$ de recursos propios y el $70 \%$ mediante un crédito a través de la CFN. La evaluación financiera identificó un VAN positivo de \$62.282,63 dólares y una TIR del 51,65\%, demostrando así la factibilidad del proyecto.

\section{Referencias bibliográficas.}

Alfaro, A. (2015). Importancia de la construcción en el mundo. Arquitectura y Construcción, 93-116.

Almoguera, J. (2013). Plan de Negocios. ESINE. Retrieved from http://www.diphuelva.es/portalweb/zonas/59/Ficheros/Plan_de_negocio.Jos\%C3\% A9_A.Almoguera.pdf

Baca, G. (2014). Evaluación de proyectos (Vol. 2). México: Editorial McGraw Hill.

BIAU. (29 de marzo de 2019). Una feria inmobiliaria presentará más de 300 proyectos de vivienda. Obtenido de https://www.elcomercio.com/construir/feria-inmobiliariapresentara-300-proyectos.html

Brock, H., \& Palmer, C. (2016). Contabilidad principios y aplicaciones. Barcelona - España: Editorial Reverté, S. A.

Daza, R. (2014). Construcción de Planes de Negocios para comercio electrónico. Medellín: Universidad EAFIT, Master's Thesis.

Eleprosh, E. (2011). Estudio Técnico. Obtenido de http://biblio3.url.edu.gt/Libros/2011/evaelePro/3.pdf

Fernández, L. (2013). Análisis de los Negocio con Excel XP. México: Pearson S.A.

Ferré, J. (2014). Los estudios de mercado. Madrid: Editorial Díaz de Santos, S.A.

Gallardo, J. (2017). Evaluación económica y financiera. México: Dirección General de Publicaciones y Fomento Editorial.

Gilli, J. (2017). Claves de la estructura organizativa. Buenos Aires: Editorial Granica.

Guzmán, F. (2016). El estudio económico financiero y la evaluación en proyectos de la industria química. Bogotá - Colombia: Universidad Nacional de Colombia.

Hamilyon, M., \& Pezo, A. (2015). Formulación y evaluación de proyectos tecnológicos empresariales aplicados. Colombia: ISBN. 
Vol. 3, N².2., p. 173-191, junio, 2019

Horngren, T., Sundem, L., \& Elliott, O. (2016). Contabilidad Financiera. México: Pearson Prentice Hall. Obtenido de http://www.ingenieria.unam.mx/industriales/descargas/documentos/catedra/apuntes _cfc.pdf

INEC. (2012). Obtenido de Encuesta nacional de ingresos y gastos de los hogares urbanos y rurales: http://www.ecuadorencifras.gob.ec//documentos/webinec/Estadisticas_Sociales/Encuesta_Nac_Ingresos_Gastos_Hogares_Urb_Rur_ENI GHU/ENIGHU-2011-2012/EnighurPresentacionRP.pdf

Jurado, J. (2016). Punto de equilibrio. Nueva York: ATRIA ESPAÑOL. Retrieved from http://www.economia.unam.mx/secss/docs/tesisfe/GomezAM/cap3.pdf

Martínez, J. (2015). Formulación y evaluación de Proyectos de Inversión. Bogotá: Instituto Superior de Coatzacoalcos. Retrieved from https://es.slideshare.net/sagjess/unidad-6estudio-administrativo-y-legal-22680464

Mundo Constructor. (2017, marzo 30). Panorama del sector de la construcción en el 2017. Quito: Mundo Constructor. Retrieved from Panorama del sector de la construcción en el 2017.

Operagb. (2017). EL Estudio de Mercado. uploads. Retrieved from http://operagb.com/wpcontent/uploads/2017/09/8448169298.pdf

Parmerle, D. (2012). Preparación del plan de marketing. Barcelona: Editorial Granica.

Rapahmell, J. (2017). Conceptos y Fundamentos de servicio, cliente y producto. Obtenido de https://www.virtuniversidad.com/greenstone/collect/negocioc/archives/HASH2ba6. dir/doc.pdf

Romo, C. (2013). Desarrollo y pasos a seguir para la Creación de un Plan de Negocios. Quito: Universidad San Francisco de Quito.

SENPLADES. (2017). Plan Nacional de Desarrollo "Toda una Vida". Quito: SENPLADES. Obtenido de PNVB 2013-2017: http://www.buenvivir.gob.ec/objetivo-3.-mejorar-lacalidad-de-vida-de-la-poblacion\#tabs3

Universidad Autónoma de México. (2011). Estudio Económico Financiero. México: Facultad de Economía. Retrieved from http://biblio3.url.edu.gt/Libros/2011/evaelePro/4.pdf

Zevallos, E. (2016). Restricciones del entorno a la competitividad empresarial en América Latina. San José - Costa Rica: FUNDES.

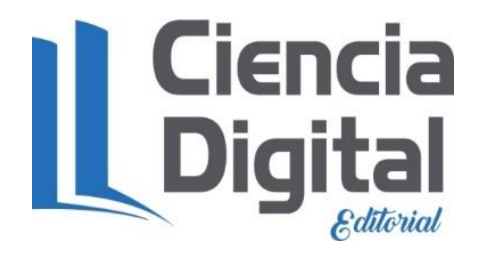




\section{PARA CITAR EL ARTÍCULO INDEXADO.}

Goercke Villarreal, J., Erazo Álvarez, J., Narváez Zurita, C., \& Quevedo Vázquez, J. (2019). Plan de negocio, proyecto constructora BellaTerra en la ciudad de Cuenca, Ecuador. Visionario $\quad$ Digital, 3(2.2), 173-191. https://doi.org/10.33262/visionariodigital.v3i2.2.629

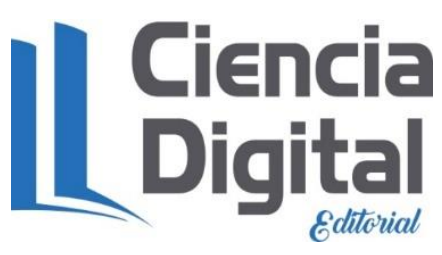

El artículo que se publica es de exclusiva responsabilidad de los autores y no necesariamente reflejan el pensamiento de la Revista Visionario Digital.

El artículo queda en propiedad de la revista y, por tanto, su publicación parcial y/o total en otro medio tiene que ser autorizado por el director de la Revista Visionario Digital.
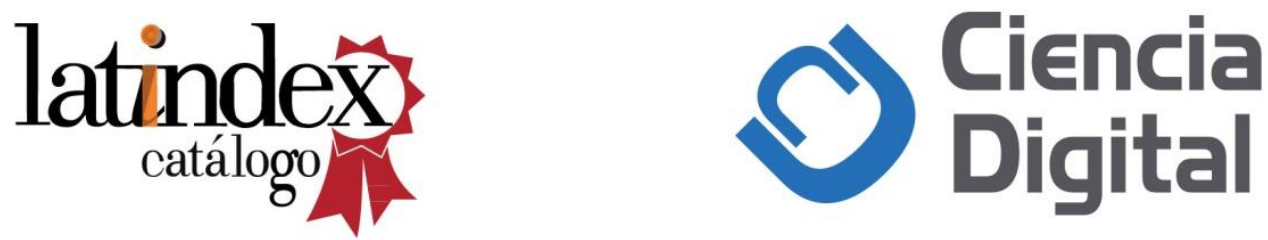